\title{
ABUNDANCES OF INTERSTELLAR MOLECULES AND THEIR FORMATION ON GRAIN SURFACES*
}

\author{
E. E. SALPETER and W. D. WATSON \\ Cornell University, Ithaca, N.Y., U.S.A.
}

\begin{abstract}
Surface phenomena on interstellar dust grains, which are relevant for molecule formation, are summarized. For various molecular species in the interstellar gas, the dependence of abundance on gas density and the degree of shielding of starlight is predicted. These predictions seem to fit with recent observations on carbon-monoxide, but there seems to be a discrepancy for formaldehyde.
\end{abstract}

\section{Introduction}

We report here briefly on two recent papers. In one (Watson and Salpeter, 1972a, hereafter referred to as WS1) surface phenomena on interstellar dust grains which are relevant for molecule formation were investigated; in the second paper (Watson and Salpeter, 1972b, hereafter referred to as WS2) we discussed destruction rates for molecules and predicted molecular abundances for the interstellar gas. This work is most relevant to 'normal' $H$ I regions, i.e., to interstellar clouds of modest density and light to moderate shielding from starlight. We first summarize the results of these two papers and then discuss comparisons with more recent observational results. The relevance of our work to the very densest 'molecular clouds' is not yet clear; but we end with some pleas to the observationalists for further measurements.

\section{Regions with Light or Moderate Shielding}

Let $n$ be the total (atomic plus molecular) number density of hydrogen in an interstellar cloud and $\tau_{v}$ the optical depth (in extinction) in the visible from the outside to the center of the cloud. The ratio of gas density (or of number-density of dust grains) to the density of attenuated UV photons from the general interstellar radiation field is approximately proportional to the parameter

$$
\xi \equiv\left(n / 100 \mathrm{~cm}^{-3}\right) e^{2,5} \tau_{v} .
$$

We consider in this section clouds where neither the shielding $\tau_{v}$ nor the density are particularly great, so that $\xi<10^{4}$ (as will be discussed subsequently, we do not understand how the molecules are prevented from condensing out of the gas into grains if $\xi>10^{4}$ ).

Most atoms and molecules (such as $\mathrm{N}, \mathrm{O}, \mathrm{CO}$, etc.) in the interstellar gas are expected to be neutral under normal ' $\mathrm{H}$ I-conditions'. For any such neutral radical or molecule hitting an interstellar dust grain, WS1 predict a high probability for it to

\footnotetext{
* Supported in part by the U.S. National Science Foundation Grant GP-26068.
} 
stick to the grain surface long enough to form some kind of a molecule by recombining with another adsorbed radical. Saturated molecules involving hydrogen $\left(\mathrm{CH}_{4}, \mathrm{NH}_{3}\right.$, $\mathrm{H}_{2} \mathrm{O}, \mathrm{H}_{2} \mathrm{~S}$, etc.) are most simply made in this manner. However, reactions between a molecule in an excited state and another molecule on the surface of the grain can also produce more complex molecules.

Some of the molecules formed on a grain surface are ejected into the gas during the process of formation, but some fraction of them remain adsorbed to the surface. An important problem is then how the adsorbed molecules are eventually ejected from the surface. The growth of a pure dust-grain is still problematical, but the case of a molecule adsorbed to a grain made of material different from the molecule is actually less uncertain. For lightly shielded clouds the UV photons with energies between about $7 \mathrm{eV}$ and $13.6 \mathrm{eV}$ ( 915 to roughly $2000 \AA$ ) are probably the most efficient ejection mechanism. WS1 estimate that this mechanism should prevent the interstellar gas from condensing out onto grains as long as $\xi$ in Equation (1) is less than about $10^{4}$. The critical value of $\xi$ for condensation is numerically uncertain by an order of magnitude or so, but experimental results from the Berkeley group (Greenberg, 1973) to be presented at this Symposium tend to corroborate at least qualitatively the efficiency of photo-ejection.

To predict molecular abundances for the interstellar gas from the formation rates on grain surfaces, estimates for the destruction rates for molecules in the gas phase are needed. For some molecular species (Solomon and Klemperer, 1972; WS2) destruction by exchange reactions (e.g., $\mathrm{OH}+\mathrm{C}^{+} \rightarrow \mathrm{CO}+\mathrm{H}^{+}$) competes with photodissociation of the molecule. In such a case (e.g., for $\mathrm{OH}$ ) the dependence of the predicted abundance on the value of $\xi$ is then of the form

$$
\frac{[\mathrm{OH}]}{[\mathrm{H}]} \approx \frac{a \xi}{1+b \breve{\xi}} .
$$

For the particular case of $\mathrm{OH}$ (and assuming the cosmic abundance ratio of $[\mathrm{O}] /[\mathrm{H}]$ $\approx 10^{-3}$ ) WS2 estimate $a / b \approx 2 \times 10^{-8}$ and $b \approx 5$. Observational data by Heiles (1971) and by Davies and Matthews (1972) indicate that $[\mathrm{OH}] /[\mathrm{H}] \approx 10^{-7}$ for $\xi \gg 0.1$ and that $[\mathrm{OH}] /[\mathrm{H}] \lesssim 2 \times 10^{-8}$ for $\xi<0.04$. The data are consistent with the functional form of Equation (2) with $a / b \approx 10^{-7}$ and $b \approx 5$. This can be considered reasonable agreement in view of the uncertainties involved.

For clouds with moderately heavy shielding, $100<\xi<10^{4}$, the atomic carbon in the gas is neutral and photons beyond $11.3 \mathrm{eV}$ energy are eleminated by its ionization edge, as well as by absorption from molecular hydrogen. This sharply decreases the photodissociation of $\mathrm{CO}$, so that much of the carbon in the cloud is in the form of gaseous CO. Other molecules have lower photodissociation thresholds. The theory thus predicts a rapid increase of the $\mathrm{CO}$-abundacne with increasing $\xi$ and (through further grain reactions) increased abundances of complex molecules containing $\mathrm{CO}$, such as formaldehyde and alcohols. Observations indeed indicate a high abundance of $\mathrm{CO}$ in dense clouds, but not a formaldehyde abundance (Heiles, 1971) as large as we predict theoretically for large $\xi$. 
The theoretical situation is complicated for carbon compounds in lightly shielded clouds, $\xi \ll 100$, where the carbon atoms in the gas are mainly ionized. In these low density clouds the dust grains are likely to be positively charged (Watson, 1972), so that the rate at which $\mathrm{C}^{+}$-ions stick to grain surfaces would be greatly reduced. The rate for surface-production of $\mathrm{CH}, \mathrm{CH}_{4}$, etc. should then also be greatly reduced. Lightly shielded clouds are the most favorable setting for the formation of $\mathrm{CH}^{+}$by radiative recombination in the gas phase from $\mathrm{C}^{+}$and $\mathrm{H}$ (Solomon and Klemperer, 1972). This gas phase reaction thus complements the surface reactions, but theoretically it is difficult to produce more complex carbon compounds in lightly shielded clouds. In particular, we seem to predict smaller formaldehyde abundances for small $\xi$ than the observations (Davies and Matthews, 1972) indicate - opposite to the situation for large $\xi$. Further detailed observations of the molecular abundances in directions of little optical extinction would be most helpful.

\section{General Discussion}

We have mainly discussed so far the formation of molecules on the surfaces of interstellar dust grains (besides the radiative recombination of $\mathrm{CH}^{+}$). One sometimes hears discussions on alternative, or 'rival', production mechanisms. We suggest here that one should also make a clear distinction between 'rival conditions' for molecule formation in interstellar space, since these conditions largely determine which of various rival production mechanisms should dominate.

We have already introduced a one-dimensional classification scheme for interstellar clouds, namely the parameter $\xi$ in Equation (1). If one attempts to equate the optical depth $\tau_{v}$ with the observed mean visual extinction for passage through a cloud or a cloud complex, additional factors that affect the physical conditions are ignored. The molecular abundances in the interior of a cloud also depend on (i) the shape of the cloud (if it has a very irregular surface, more photons can penetrate into the interior), (ii) whether young stars are imbedded in the cloud complex, (iii) whether turbulence can produce shock-wave heating (Bar-Nun et al., 1970) or can 'turn the cloud inside out' within a relaxation time for condensation of gas molecules onto grains, etc. Our first plea to observers is to measure systematically abundances for various molecules in clouds with different density and shielding. Our second plea is to measure these abundances for spatial fine-structure, i.e., determine the variation of molecular abundances within a single cloudcomplex.

For a cloud-complex with moderate shielding, $\xi \sim \xi_{0} \ll 10^{4}$, the theory would predict a greater abundance for many molecules in the deep interior where the shielding is greatest. Observational abundance ratios from the center to the edge of such a cloud will be welcome. Theory predicts the opposite difficulty for a very dense cloud complex with $\xi \gg 10^{4}$. There is no difficulty in making molecules in the most shielded regions but it seems that they should be condensed out as grain mantles. In this case molecules in the gas phase will be more abundant near the boundaries of the cloud complex. Thus it will be particularly interesting to study observationally whether 
there really is a correlation between abundances of some molecules and local $\mathrm{H}$ IIregions inside a large cloud complex. If the complex is highly shielded from the average interstellar radiation field but has some hot stars embedded in it, there should be a 'spherical shell' just outside the 'Strömgren sphere' surrounding each star where the hydrogen is neutral but some photons from the star between $\approx 7 \mathrm{eV}$ and $13.6 \mathrm{eV}$ are present. These photons could prevent the molecules from condensing out onto grains without destroying all the molecules. Hence molecular abundances could peak in such a 'spherical shell' around each star.

Molecule formation in the very dense, contracting nebula surrounding a proto-star (a generalized 'solar nebula') has been discussed by various authors (Herbig, 1971; Anders, 1972; Sagan, 1972). Molecule formation in the atmosphere of an existing, cool star will be discussed at this Symposium (Shimizu, 1973). There is certainly no difficulty in making molecules under these high-density conditions. It has been pointed out (Salpeter, 1971) that an 'average atom' passes through such conditions only about once in the life-time of the Galaxy, whereas it collides with dust grains a few hundred times during this period. For many molecules under 'average conditions' one would expect formation on grain surfaces to dominate, but this certainly will not be universally true and it will be useful to watch for characteristic differences in the molecular abundances predicted for formation under the various conditions.

For clouds with light and moderate shielding we have stressed the formation of molecules on grain surfaces (plus radiative recombination of $\mathrm{CH}^{+}$), but we are really dealing with a cycle for which the UV photons (energies of about $10 \mathrm{eV}$ ) provide the 'driving force', i.e., the source of energy and entropy. These photons not only eject molecules from grains and destroy the molecules directly, but by maintaining an appreciable abundance of atoms or radicals in the gas they also insure indirectly that many electron volts of excitation energy are available for the surface reactions required to form some molecules. As a consequence, the ratio of the rates of formation of two different molecules with comparable numbers of constituent $\mathrm{C}, \mathrm{N}$, and $\mathrm{O}$-atoms depends on the properties of the surface and does not involve a Boltzmann factor. Although it is difficult to predict this ratio, it might be near unity for some molecules even though their binding energies differ by a few eV. In contrast these abundance ratios exhibit a Boltzmann factor involving the difference in binding energies for molecule production in stellar atmospheres (Shimizu, 1973). For example, production in a stellar atmosphere with a temperature of $\approx 2500 \mathrm{~K}$ leads to a ratio $\approx 10^{4}$ when the binding energies differ by $2 \mathrm{eV}$ - a representative energy difference (the actual observed ratio may be further increased by rearrangement collisions as the gas flows away from the star and cools).

In the 'solar-type nebula' surrounding a proto-star, reactions on grain-surfaces are also considered, but the details are drastically different because UV photons are absent and the kinetic temperature is a few hundred degrees. Instead of a helter-skelter production of many different molecules, one relies on a chain of catalytic reactions. This will single out a subset of all possible types of molecules, somewhat as in meteorites and in terrestrial Fischer-Tropsch reactions (Anders, 1972). 
Finally we emphasize that the major uncertainty in understanding the large molecule abundances in dense clouds does not seem to be how and where the molecules are formed, but why they are not condensed onto the dust grains that cause the observed extinction.

\section{References}

Anders, E.: 1972, in Proceedings of the Symposium on Interstellar Molecules (Charlottesville, Virginia, October 1971), in press.

Bar-Nun, A., Bar-Nun, N., Bauer, S., and Sagan, C.: 1970, Science 168, 470.

Davies, R. D. and Matthews, H. E.: 1972, Monthly Notices Roy. Astron. Soc. 145, 249.

Greenberg, L. T.: 1973, this volume, p. 413.

Heiles, C.: 1971, Ann. Rev. Astron. Astrophys. 9, 293.

Herbig, G. H.: 1971, in C. de Jager (ed.), Highlights of Astronomy, D. Reidel, Dordrecht.

Sagan, C.: 1972, in Proceedings of the Symposium on Interstellar Molecules (Charlottesville, Virginia, October 1971), in press.

Salpeter, E. E.: 1971, in C. de Jager (ed.), Highlights of Astronomy, D. Reidel, Dordrecht.

Shimizu, M.: 1973, this volume, p. 405.

Solomon, P. M. and Klemperer, W.: 1972, Astrophys. J. 178, 389.

Watson, W. D.: 1972, Astrophys. J. 176, 103 and 271.

Watson, W. D. and Salpeter, E. E.: 1972a, Astrophys. J. 174, 321 (WS1).

Watson, W. D. and Salpeter, E. E.: 1972b, Astrophys. J. 175, 659 (WS2). 